\title{
ON THE CLASSIFICATION OF TRANSITIVE EFFECTIVE ACTIONS ON STIEFEL MANIFOLDS
}

\author{
BY \\ WU-YI HSIANG AND J. C. SU( $\left.{ }^{1}\right)$
}

Introduction. Let $G$ be a compact connected Lie group and $H$ a closed subgroup of $G$. Then the coset space $G / H$ is a smooth manifold with $G$ acting transitively as translations. It is easy to see that the natural action of $G$ on $G / H$ is effective if and only if $H$ contains no nontrivial normal subgroup of $G$. For a given compact homogeneous space $M=G / H$, one might ask whether there are any other (differentiably nonequivalent) transitive effective actions on $M$ ? And furthermore, what are all the possible nonequivalent transitive effective actions on $M$ ? In the special case that $M$ is a sphere, the above classification problem has been completely solved by the successive efforts of Montgomery and Samelson [10], Borel [1], and Poncét [11]. The purpose of this paper is to continue, along this direction, to classify the transitive effective actions on the Stiefel manifolds.

Let $V_{n, k}$ be the Stiefel manifold of orthonormal $(n-k)$-frámes in the euclidean $n$-space. If we consider $V_{n, k}$ as a subset of the space of $n \times(n-k)$ matrices, then $\mathrm{SO}(n)$ acts on $V_{n, k}$ by matrix multiplication from the left and $\mathrm{SO}(n-k)$ acts on $V_{n, k}$ by matrix multiplication from the right. Suppose $G$ is any compact Lie group such that $\mathrm{SO}(n) \subset G \subset \mathrm{SO}(n) \times \mathrm{SO}(n-k)$, then it is easy to see that $G$ acts on $V_{n, k}$ transitively (and effectively in many cases). Our main result is that:

"For many values of $n$ and $k$, every transitive effective action on $V_{n, k}$ is differentiably equivalent to one of the above examples."

Parallel results are also proved for the complex and symplectic Stiefel manifolds.

Technically, the most difficult part of the proof is to show that any transitive group $G$ on $V_{n, k}$ contains a simple normal subgroup $G_{1}$ that already acts transitively! In the case of transitive groups on spheres, the above fact is an easy consequence of the particularly simple structure of the cohomology group of spheres [10], which is no longer available in our case. For this purpose we introduce the concept of irreducible transitive action, namely, $G$ is said to be an irreducible transitive group on $M$ if there is no proper normal subgroup of $G$ that is already transitive on $M$. With the help of a cohomological criterion of irreducible transitivity, the uniqueness of irreducible transitive effective action on a Stiefel manifold is established

Received by the editors July $18,1966$.

(1) Research supported in part by NSF grant GP-2440; the second named author also received support from NSF grant GP-4125. The authors acknowledge with pleasure many suggestions from Wu-chung Hsiang. 
through two complementary steps that we shall state as decomposition theorem and nondecomposition theorem. Roughly speaking, they are as follows:

(i) Under quite general conditions on the cohomology of the given homogeneous space $M$, if an irreducible transitive group on $M$ is nonsimple, then $M$ decomposes into the product of two homogeneous spaces.

(ii) For most Stiefel manifolds, such decompositions are impossible.

We would like to point out that if $M=G / H$, where $H \subset G$ is of maximum rank, the decomposition theorem is a trivial consequence of the maximum torus theorem. Thus in a technical sense, the Stiefel manifolds pose a quite different problem to other spaces, say, like complex projective spaces of Grassmanian manifolds. Indeed, this is the reason our choice is made.

The ultimate question one would like to settle is of course the following: Given a homogeneous space $M=G / H$ with $G$ a simple compact connected Lie group, should the natural action of $G$, allowing perhaps a few exceptions, be the only irreducible transitive action on $M$ ?

1. Preliminaries. By a homogeneous space, we shall mean a left coset space $M=G / H$, where $G$ is a compact connected Lie group and $H \subset G$ a closed subgroup. We shall moreover assume that $M$ is simply connected so that $H$ is also connected. In terms of transformation group, $G$ acts transitively on $M$ with $H$ as the isotropy subgroup at a certain point of $M$. We shall use the triple notation $(G, H ; M)$ to denote $M=G / H$. The homogeneous spaces that we shall be concerned with in this paper are: The real Stiefel manifold $V_{n . k}$ of $(n-k)$-orthonormal frames in real $n$-space $R^{n}$, the complex Stiefel manifold $W_{n, k}$ of $(n-k)$-orthonormal frames in complex $n$-space $C^{n}$, and the symplectic Stiefel manifold $X_{n, k}$ of $(n-k)$-orthonormal frames in the quaternion $n$-space $Q^{n}$. As is well known, we have then the triples $\left(\mathrm{SO}(n), \mathrm{SO}(k) ; V_{n, k}\right),\left(\mathrm{SU}(n), \mathrm{SU}(k) ; W_{n, k}\right)$ and $\left(\mathrm{Sp}(n), \mathrm{Sp}(k) ; X_{n, k}\right)$, where $\mathrm{SO}($ ), SU( ), and $\mathrm{Sp}(\quad)$ denote the special orthogonal, the special unitary, and the symplectic groups of appropriate dimensions. We shall refer to them as standard actions. In case $k=n-1$, these manifolds become spheres of various dimensions. Since the question of classifying transitive actions on spheres has already been done by various authors (see introduction), we shall assume throughout the rest of this paper that $k \leqq n-2$.

In the study of a homogeneous space $(G, H ; M)$, it is useful to find topological means to determine the rank difference $\operatorname{Rk}(G)-\operatorname{Rk}(H)$ (where $\operatorname{Rk}(G)$ means the rank of $G$ ). For instance, it is well known that $\operatorname{Rk}(G)=\operatorname{Rk}(H)$ if and only if the Euler characteristic of $M$ is nonvanishing. In general, we propose to define a rank for a homogeneous space $M$, to be denoted by $\operatorname{Rk}(M)$, as the alternate sum $-\sum_{i=0}^{\infty}(-1)^{i} \operatorname{dim}\left(\pi_{i}(M) \otimes Q\right)$, where $\pi_{i}(M)$ is the $i$ th homotopy group of $M$ and $Q$ the field as rationals.

Proposition 1.1. Let $(G, H ; M)$ be a homogeneous space, then $\operatorname{Rk}(M)$ is well defined and is equal to $\operatorname{Rk}(G)-\operatorname{Rk}(H)$. 
Proof. According to Milnor and Moore [8, p. 263], for a compact connected Lie group $G,-\sum_{i=0}^{\infty}(-1)^{i} \operatorname{dim}\left(\pi_{i}(G) \otimes Q\right)$ is always well defined and is equal to the rank of $G$. Our proposition follows immediately from the homotopy exact sequence of the fibration $H \rightarrow G \rightarrow M$.

The above simple observation is sometimes quite useful in analyzing the Serre spectral sequence associated with the fibration $H \rightarrow G \rightarrow M$, which we shall spell out explicitly next. Recall that for a compact connected Lie group $G$, the rational cohomology $H^{*}(G ; Q)$ is a connected Hopf algebra. Let $P(G)$ be its graded vector space of primitive elements. Then it is well known that $H^{*}(G ; Q)=\Lambda(P(G))$ as a Hopf algebra, where $\Lambda(P(G))$ is the exterior algebra of $P(G)$ endowed with the usual diagonal map. Let us call a homogeneous space $M$ a Hopf homogeneous space if (i) $H^{*}(M ; Q)$ is an exterior algebra of the form $\Lambda(P(M))$, where $P(M)$ is a graded vector space having a homogeneous base of odd degrees, and (ii) $\operatorname{dim}(P(M))$ $=\operatorname{Rk}(M)$.

Proposition 1.2. Let $(G, H ; M)$ be a Hopf homogeneous space, then the Serre spectral sequence associated with the fibration

$$
H \stackrel{i}{\longrightarrow} G \stackrel{\pi}{\longrightarrow} M
$$

(rational coefficient) is trivial. In particular, the sequence

$$
0 \longrightarrow P(M) \stackrel{\pi^{*}}{\longrightarrow} P(G) \stackrel{i^{*}}{\longrightarrow} P(H) \longrightarrow 0
$$

is exact.

Proof. In the Serre spectral sequence, we have $E_{2}=H^{*}(M ; Q) \otimes H^{*}(H ; Q)$. By condition (ii), $\operatorname{dim} H^{*}(M ; Q)=2^{\mathrm{Rk}(M)}$. Hence $\operatorname{dim} E_{2}=2^{\mathrm{Rk}(M)+\mathrm{Rk}(H)}=2^{\mathrm{Rk}(G)}$ in view of Proposition 1.1. On the other hand, $\operatorname{dim} E_{\infty}=\operatorname{dim} H^{*}(G ; Q)=2^{\mathrm{Rk}(G)}$. It follows that the spectral sequence is trivial. Now in general, $\operatorname{Im} \pi^{*} \subset H^{*}(G ; Q)$ is always a Hopf subalgebra [2]. As $\pi^{*}$ is a monomorphism in our case, $H^{*}(M ; Q)$ can be viewed as a Hopf algebra so that $\pi^{*}$ becomes a Hopf algebra homomorphism (this justifies our terminology), giving the exactness of the sequence stated.

The rational cohomology of all the Stiefel manifolds are exterior algebras. In the table below we list the degrees and number of generators of these algebras [2].

$$
\begin{aligned}
& \begin{array}{llll}
M & P(M) & \operatorname{dim} P(M) & \operatorname{Rk}(M)
\end{array}
\end{aligned}
$$

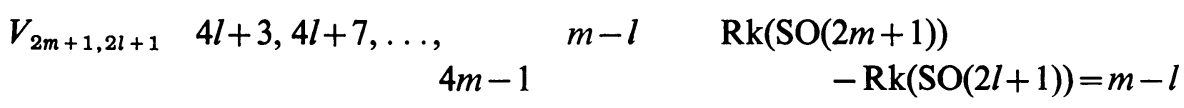

$$
\begin{aligned}
& V_{2 m, 2 l+1} \quad 4 l+3,4 l+7, \ldots, \quad m-l \quad \operatorname{Rk}(\mathrm{SO}(2 m)) \\
& 4 m-5,2 m-1 \quad-\mathrm{Rk}(\mathrm{SO}(2 l+1))=m-l \\
& V_{2 m+1,2 l} \quad 2 l, 4 l+3,4 l+7, \ldots, \quad m-l+1 \quad \operatorname{Rk}(\operatorname{SO}(2 m+1)) \\
& 4 m-1 \quad-\operatorname{Rk}(\operatorname{SO}(2 l))=m-l
\end{aligned}
$$




$$
\begin{array}{cccc}
V_{2 m, 2 l} & 2 l, 4 l+3,4 l+7, \ldots, & m-l+1 & \mathrm{Rk}(\mathrm{SO}(2 m)) \\
& 4 m-5,2 m-1 & & -\operatorname{Rk}(\operatorname{SO}(2 l))=m-l \\
W_{n, k} & 2 k+1,2 k+3, \ldots, & n-k & \operatorname{Rk}(\mathrm{U}(n))-\operatorname{Rk}(\mathrm{U}(k))=n-k \\
& 4 k-1 & & \\
X_{n, k} & 4 k+3,4 k+7, \ldots, & n-k & \operatorname{Rk}(\operatorname{Sp}(n))-\operatorname{Rk}(\operatorname{Sp}(k))=n-k \\
& & &
\end{array}
$$

With the exception of real Stiefel manifold $V_{n, k}, k$ even, we see that all other homogeneous spaces we shall be concerned with, namely $V_{n, k}$ with $k$ odd, $W_{n, k}$ and $X_{n, k}$ are Hopf homogeneous spaces for which Proposition 1.2 is applicable. We thus need a slightly different version of the above proposition to take care of $V_{n, 2 l}$. Recall that $P\left(V_{n, 2 l}\right)$ has a generator $v_{2 l}$ of even degree $2 l$ and this is a generator of lowest degree. Write $H^{*}(H ; Q)=\Lambda\left(x_{1}, \ldots, x_{r}\right), r=\operatorname{Rk}(H)$, we have

Proposition $1.2^{\prime}$. Let $\left(G, H ; V_{n, 2 l}\right)$ be a transitive action, then the following is true:

(i) The kernel of $\pi^{*}: H^{*}\left(V_{n, 2 l} ; Q\right) \rightarrow H^{*}(G ; Q)$ is the ideal generated by $v_{2 l}$.

(ii) There is a generator in $H^{*}(H ; Q)$, say $x_{r}$, of degree $2 l-1$, which corresponds to $v_{2 l}$ under transgression. All other generators of $H^{*}(H ; Q)$ have zero transgression.

(iii) There is a generator in $H^{*}(G ; Q)$ of degree $4 l-1$, to be denoted by $y_{4 l-1}$, such that the kernel of $i^{*}: H^{*}(G ; Q) \rightarrow H^{*}(H ; Q)$ is the ideal generated by $y_{4 l-1}$ and $\operatorname{Im} \pi^{*}$.

(iv) The image of $i^{*}$ is the subalgebra generated by $x_{1}, \ldots, x_{r-1}$, i.e., the set of generators of $H^{*}(H ; Q)$ with the special element $x_{r}$ deleted.

(v) Define $P^{\prime}\left(V_{n, 2 l}\right)=P\left(V_{n, 2 l}\right) /\left\langle v_{2 l}\right\rangle\left(P\left(V_{n, 2 l}\right)\right)$ modulo the subspace spanned by $\left(v_{2 l}\right), P^{\prime}(G)=P(G) \mid\left\langle y_{4 l-1}\right\rangle$ and $P^{\prime}(H)=\left\langle x_{1}, \ldots, x_{r-1}\right\rangle \subset P(H)$. Then the sequence

$$
0 \longrightarrow P^{\prime}\left(V_{n, 2 l}\right) \stackrel{\pi^{*}}{\longrightarrow} P^{\prime}(G) \stackrel{i^{*}}{\longrightarrow} P^{\prime}(H) \longrightarrow 0
$$

is exact.

For the proof, we shall only mention that we have used the fact that $\operatorname{Im} \pi^{*}$ is generated by $\operatorname{Im} \pi^{*} \cap P(G)$ [2, Proposition 21.1]. This plus the fact that $v_{2 l}$ has lowest degree in $P\left(V_{n, 2 l}\right)$ implies that $\pi^{*}\left(v_{2 l}\right)=0$. The rest follow from the known structure of $H^{*}\left(V_{n, 2 l} ; Q\right)$ and Proposition 1.1.

Very often in the sequel, we shall find it necessary to pay special attention to $V_{n, 2 l}$ owing to the fact that it has a generator of even degree in $H^{*}\left(V_{n, 2 l} ; Q\right)$. As illustrated by the above proposition, it presents a slight complication but no fundamental difficulty. We shall therefore adopt the philosophy of stating a corresponding proposition, if necessary, but with proof omitted.

Definition 1.3. A transitive action $(G, H ; M)$ is said to be irreducible if the action restricted to any proper connected closed normal subgroup of $G$ ceases to be transitive. 
For a subgroup $G_{0} \subset G$, let $j_{0}: G_{0} \rightarrow G$ be the inclusion and $\pi_{0}: G_{0} \rightarrow M$ the composition

$$
G_{0} \stackrel{j_{0}}{\longrightarrow} G \stackrel{\pi}{\longrightarrow} M
$$

Proposition 1.4. Let $(G, H ; M)$ be a Hopf homogeneous space. Then the action is irreducible if and only if for every proper connected closed normal subgroup $G_{0} \subset G$, $\pi_{0}^{*}: P(M) \rightarrow P\left(G_{0}\right)$ is not a monomorphism.

Proof. The "if" part is contained in Proposition 1.2. For the "only if" part, suppose that $G_{0} \subset G$ is a proper connected closed normal subgroup such that $\pi_{0}^{*}$ is a monomorphism. We shall prove that $G_{0}$ acts transitively on $M$, i.e., the orbit $G_{0} / G_{0} \cap H \subset M$ is all of $M$. Let $p: G \rightarrow G / G_{0}$ be the projection and $i_{0}: H \rightarrow G / G_{0}$ the composition

$$
H \stackrel{i}{\longrightarrow} G \stackrel{p}{\longrightarrow} G / G_{0}
$$

We have the following commutative diagram

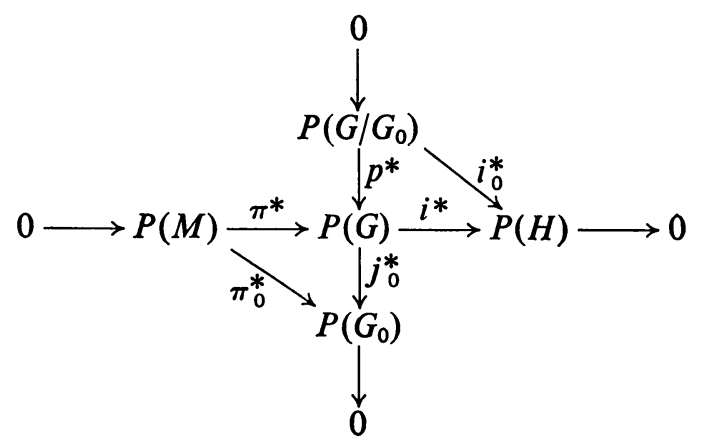

with exact row and column (because $G / G_{0}$ is necessarily Hopf homogeneous). If $\pi_{0}^{*}$ is a monomorphism, so is $i_{0}^{*}$ as an easy diagram chasing would show. Since $H^{*}\left(G / G_{0} ; Q\right)$ and $H^{*}(H ; Q)$ are both connected Hopf algebras, it follows from [8] that $i_{0}^{*}: H^{*}\left(G / G_{0} ; Q\right) \rightarrow H^{*}(H ; Q)$ is also a monomorphism. But this could happen only if $i_{0}(H)=G / G_{0}$ for dimension reason. Thus the groups $G / G_{0}$ and $H / H \cap G_{0}$ are isomorphic. This gives the relation

$$
\begin{aligned}
\operatorname{dim} G_{0} / H \cap G_{0} & =\operatorname{dim} G_{0}-\operatorname{dim} H \cap G_{0} \\
& =\operatorname{dim} G-\operatorname{dim} H \\
& =\operatorname{dim} M,
\end{aligned}
$$

or $G_{0} / H \cap G_{0}=M$.

For a triple $\left(G, H ; V_{n, 2 l}\right)$, we shall call a normal subgroup $G_{0} \subset G$ admissible if $j_{0}^{*}\left(y_{4 l-1}\right) \neq 0$ (see Proposition 1.2'). In such a case, define $P^{\prime}\left(G_{0}\right)=P\left(G_{0}\right) /\left\langle j_{0}^{*}\left(y_{4 l-1}\right)\right\rangle$.

Proposition 1.4'. A transitive action $\left(G, H ; V_{n, 2 l}\right)$ is irreducible if and only if 
for every proper connected closed admissible normal subgroup $G_{0} \subset G, \pi_{0}^{*}: P^{\prime}\left(V_{n, 2 l}\right)$ $\rightarrow P^{\prime}\left(G_{0}\right)$ is not a monomorphism.

2. A decomposition theorem for irreducible actions. Recall that given a compact connected Lie group $G$, one can always write $G=T^{r} \times G_{1} \times \cdots \times G_{s} / N$, where $T^{r}$ is a $r$-dimensional torus, $G_{i}, i=1,2, \ldots, s$, are simply connected simple Lie groups, and $N \subset T^{r} \times G_{1} \times \cdots \times G_{s}$ is a finite normal subgroup. Let $\tilde{G}=T^{r} \times G_{1} \times \cdots \times G_{s}$, $p: \widetilde{G} \rightarrow G$ the canonical projection. If $(G, H ; M)$ is a transitive action, $\widetilde{G}$ acts transitively on $M$ in a natural way with isotropy subgroup $\tilde{H}=p^{-1}(H)$ so we have the triple $(\tilde{G}, \tilde{H} ; M)$, which we may call the lifted action. In what follows, we shall simply use the notation $(G, H ; M) \sim(\tilde{G}, \tilde{H} ; M)$ without further explanation. Simple Lie groups are exhausted up to local isomorphism by four series $A_{n}, B_{n}$, $C_{n}, D_{n}$ and five exceptional Lie groups: $G_{2}, F_{4}, E_{6}, E_{7}$ and $E_{8}$. Simple Lie groups belonging to the series $A_{n}, B_{n}, C_{n}$, and $D_{n}$ are called classical. Their (unique) simply connected representatives are $\operatorname{SU}(n+1)\left(A_{n}\right)$, the spinor group $\operatorname{Spin}(2 n+1)\left(B_{n}\right)$, $\operatorname{Sp}(n)\left(C_{n}\right)$, and $\operatorname{Spin}(2 n)\left(D_{n}\right)$. For reference, we list below the degree and number of primitive generators of the rational cohomology of simple Lie groups

$$
\begin{aligned}
& A_{n}: 3,5,7, \ldots, 2 n+1 \\
& B_{n}: 3,7,11, \ldots, 4 n-1 \\
& C_{n}: 3,7,11, \ldots, 4 n-1 \\
& D_{n}: 3,7,11, \ldots, 4 n-5,2 n-1 \\
& G_{2}: 3,11 \\
& F_{4}: 3,11,15,23 \\
& E_{6}: 3,9,11,15,17,23 \\
& E_{7}: 3,11,15,19,23,27,35 \\
& E_{8}: 3,15,23,27,35,39,47,59 .
\end{aligned}
$$

Let $M$ be a Hopf homogeneous space. Let $l(M)$ and $u(M)$ be the lowest and highest nonvanishing degrees of $P(M)$. The purpose of this section is to prove the following:

TheOREM 2.1. Let $(G, H ; M)$ be an irreducible action on a Hopf homogeneous space $M$ and $(\tilde{G}, \tilde{H} ; M) \sim(G, H ; M)$ the lifted action. Suppose that $l(M)>59$ and $2 l(M)>u(M)+11$. Suppose moreover that $M$ is 2-connected. Then $\tilde{G}=G_{1} \times \cdots \times G_{s}$ and $\tilde{H}=H_{1} \times \cdots \times H_{s}$ with $H_{i} \subset G_{i}$, where $G_{i}$ and $H_{i}, i=1,2, \ldots, s$, are all simply connected simple classical groups. In particular, $M$ decomposes into a product $G_{1} / H_{1} \times \cdots \times G_{s} / H_{s}$ of homogeneous spaces.

We first assert that $G$ is semisimple. That is, write $\tilde{G}=T^{r} \times G_{1} \times \cdots \times G_{s}$. We wish to show that $r=0$. It is clear that $(\tilde{G}, \tilde{H} ; M)$ is still irreducible. Now suppose that $r>0$. Referring back to the diagram of Proposition 1.4, take $G_{0}=G_{1} \times \cdots \times G_{s}$. We see that anything in ker $\pi_{0}^{*}$ must have degree 1 because everything in $P\left(T^{r}\right)$ has degree 1 . As $l(M)>1$, there is nothing in $P(M)$ of degree 1 . Hence $\pi_{0}^{*}$ is a monomorphism, contradiction. Similar argument also shows that none of the factor $G_{i}, i=1,2, \ldots, s$, could be exceptional in view of the condition $l(M)>59$ and the 
fact that none of the exceptional group has a primitive generator of degree $>59$. Thus $\tilde{G}=G_{1} \times \cdots \times G_{s}$ is a product of simply connected simple classical groups. In particular, $\widetilde{G}$ is simply connected. Since $M$ is assumed to be 2-connected, $\tilde{H}$ is simply connected. Hence we can write $\tilde{H}=H_{1} \times \cdots \times H_{s^{\prime}}$, where $H_{i}, i=1,2, \ldots, s^{\prime}$, are simply connected simple Lie groups. Observe that $\operatorname{dim} P_{3}(\tilde{G})=\operatorname{dim} P_{7}(\tilde{G})=s$ $\left(P_{i}(G)=\right.$ subspace of $P(G)$ of elements of homogeneous degree $\left.i\right)$. For $P_{3}(\widetilde{G})$, this is clear after a glance at the reference table. For $P_{7}(\tilde{G})$, this is because the irreducibility implies that every $G_{i}$ has a nonvanishing element in $P\left(G_{i}\right)$ of degree $>l(M)$. Now we have $\operatorname{dim} P_{3}(\tilde{G})=\operatorname{dim} P_{3}(\tilde{H})$ and $\operatorname{dim} P_{7}(\tilde{G})=\operatorname{dim} P_{7}(\tilde{H})$, again because $l(M)>59$. The first equality gives us $s^{\prime}=s$. The second equality shows that $\tilde{H}$ contains no exceptional group because of the happy fact that none of the exceptional group has a primitive generator of degree 7. Thus far, we have shown that $\widetilde{G}=G_{1} \times \cdots \times G_{s}$ and $\tilde{H}=H_{1} \times \cdots \times H_{s}$ both decompose into product of simply connected classical groups with same number of factors. The issue remains to be settled is the way of imbedding. Namely, how do we show that $H_{i} \subset G_{i}$ (after suitable change of indices of course). This is the question of representations of classical groups into classical groups, so we shall recall some basic facts there. There are the standard imbeddings $\mathrm{SO}(k) \subset \mathrm{SO}(n)(\operatorname{Spin}(k) \subset \operatorname{Spin}(n)), \quad \mathrm{SU}(k)$ $\subset \mathrm{SU}(n)$ and $\mathrm{Sp}(k) \subset \mathrm{Sp}(n)$ induced from $R^{k} \subset R^{n}, C^{k} \subset C^{n}$, and $Q^{k} \subset Q^{n}$ respectively. There are also the imbeddings $\mathrm{SO}(n) \subset \mathrm{SU}(n) \subset \mathrm{SO}(2 n)$ (and the homomorphism $\operatorname{Spin}(n) \rightarrow \mathrm{SU}(n) \rightarrow \operatorname{Spin}(2 n)$ induced) and $\mathrm{SU}(n) \subset \mathrm{Sp}(n) \subset \mathrm{SU}(2 n)$. We shall indiscriminately call a homomorphism $\phi$ between classical groups "standard" if $\phi$ is conjugate to one of the homomorphisms described above or their compositions. Given an irreducible complex representation $\phi: G \rightarrow \mathrm{SU}(n), \operatorname{Im} \phi \subset \mathrm{SO}(n)$ $\subset \mathrm{SO}(n)(\operatorname{Im} \phi \subset \mathrm{Sp}(n / 2) \subset \mathrm{SU}(n))$ if and only if $\phi$ keeps a nondegenerate symmetric (nondegenerate skew symmetric) bilinear form on $C^{n}$ invariant. Or equivalently, $\phi$ is real (symplectic) if and only if $\phi^{*}=\phi-\phi^{*}$ is the complex conjugate of $\phi$-and the symmetric tensor product $S^{2} \phi$ (the second exterior power $\Lambda^{2} \phi$ ) contains a trivial representation [6]. In case $\phi$ is irreducible and $\phi^{*} \neq \phi$ (nonequivalent), then $\phi+\phi^{*}$ is real as well as symplectic. With the help of Weyl character formula [5], the complex irreducible representations of low dimension of classical groups are easily identified. Together with the facts cited above, one can see by patient checking that the following is true.

(2.2) The only nontrivial homomorphism of $G=\operatorname{Spin}(k), \operatorname{SU}(k)$ or $\operatorname{Sp}(k / 2)$ into $\mathrm{SU}(n)$ in the range $k \geqq 10, k \leqq n$, and $2 k>n$ is the standard homomorphism $G \rightarrow \mathrm{SU}(k)$ $\subset \mathrm{SU}(n)$ plus its complex conjugate if $G=\mathrm{SU}(k)$.

(2.3) The only nontrivial homomorphism of $G=\operatorname{Spin}(k), \mathrm{SU}(k / 2)$, or $\mathrm{Sp}(k / 4)$ into $\operatorname{Spin}(n)$ in the range $k \geqq 16, k \leqq n$ and $2 k>n$ is the standard homomorphism $G \rightarrow$ $\operatorname{Spin}(k) \subset \operatorname{Spin}(n)$.

(2.4) The only nontrivial homomorphism of $G=\operatorname{Spin}(k), \mathrm{SU}(k)$, or $\mathrm{Sp}(k)$ into $\mathrm{Sp}(n)$ in the range $k \geqq 10, k \leqq n$ and $2 k>n$ is the standard homomorphism $G \rightarrow$ $\mathrm{Sp}(k) \subset \mathrm{Sp}(n)$. 
(2.5) In each of the cases covered by (2.2) through (2.4), the centralizer of the image of $G$ in $\mathrm{SU}(n), \operatorname{Spin}(n)$ or $\mathrm{Sp}(n)$ is locally isomorphic to $G^{\prime} \times H$, where $\operatorname{Rk}(H) \leqq 1$ and $G^{\prime}=\mathrm{SU}(n-k), \operatorname{Spin}(n-k)$ or $\mathrm{Sp}(n-k)$ respectively.

We proceed to establish two lemmas relating representation and cohomology. For a compact connected Lie group $G$, we shall use $n(G)$ to denote the top nonvanishing degree of $P(G)$.

LEMMA 2.6. Let $K$ and $G$ be simply connected classical groups with $n(K) \geqq 32$. If $\phi: K \rightarrow G$ is a nontrivial homomorphism, then $n(K) \leqq n(G)$. Moreover $n(K)=n(G)$ only when

(i) $\phi$ is an isomorphism,

(ii) $K=\operatorname{Spin}(2 k-1), G=\operatorname{Spin}(2 k), k \geqq 10$, and $\phi: \operatorname{Spin}(2 k-1) \rightarrow \operatorname{Spin}(2 k)$ is standard,

(iii) $K=\mathrm{Sp}(k), G=\mathrm{SU}(2 k) k \geqq 8$, and $\phi: \mathrm{Sp}(k) \rightarrow \mathrm{SU}(2 k)$ is standard.

Proof. We just check every possible case. Take for example $K=\operatorname{Spin}(2 k-1)$ and $G=\operatorname{Spin}(2 m)$. We must have $2 k-1 \leqq 2 m-1$ in order that $\phi$ can be nontrivial. So $n(K)=4 k-5 \leqq 4 m-5=n(G)$. To have $n(K)=n(G)$, we must have $k=m$ or $K=\operatorname{Spin}(2 k-1)$ and $G=\operatorname{Spin}(2 k)$. Since $n(K)=4 k-5 \geqq 32,2 k-1 \geqq 19$. So we are in the right range stated in (2.4) and therefore $\phi$ must be standard.

Lemma 2.7. Let $K_{1}, K_{2}$, and $G$ be simply connected classical groups with $n\left(K_{1}\right)$, $n\left(K_{2}\right)$ and $n(G) \geqq 32$. Let $\phi: K_{1} \times K_{2} \rightarrow G$ be a homomorphism and $\phi_{i}, i=1,2$ its restriction on $K_{i}, i=1$, 2. If $n\left(K_{1}\right)+n\left(K_{2}\right)>n(G)+3$, then either $\phi_{1}$ or $\phi_{2}$ is trivial.

Proof. Again we do it by case checking. Suppose that both $\phi_{1}$ and $\phi_{2}$ are nontrivial and $n\left(K_{1}\right) \geqq n\left(K_{2}\right)$. It is then easily seen that $\phi_{1}: K_{1} \rightarrow G$ is among one of the cases listed in (2.2) through (2.4). Say $K_{1}=\mathrm{SU}\left(n\left(K_{1}\right)+1 / 2\right), G=\mathrm{SU}(n(G)+1 / 2)$ and $\phi_{1}$ is standard. Now $\phi_{2}\left(K_{2}\right)$ must be in the centralizer of $\phi_{1}\left(K_{1}\right)$ in $G$. We thus obtain a nontrivial homomorphism $\phi_{2}: K_{2} \rightarrow \mathrm{SU}(m)$, where $m=\left(n(G)-n\left(K_{1}\right)\right) / 2$. As $n(\mathrm{SU}(m))=2 m-1=n(G)-n\left(K_{1}\right)-1<n\left(K_{2}\right)-4<n\left(K_{2}\right)$, this contradicts Lemma 2.6.

With this much disposed of, we shall now continue the proof of Theorem 2.1. So far, we have only shown that $\tilde{G}=G_{1} \times \cdots \times G_{s}$ and $\tilde{H}=H_{1} \times \cdots \times H_{s}$ both decompose into $s$ factors of simply connected simple classical groups. Let $p_{i}: \widetilde{G} \rightarrow G_{i}$ be the $i$ th projection and $i_{i}: H_{i} \rightarrow \tilde{H}$ the $i$ th inclusion. Also denote the compositions

$$
\tilde{H} \stackrel{i}{\longrightarrow} \tilde{G} \stackrel{p_{i}}{\longrightarrow} G_{i} \text { and } H_{j} \stackrel{i_{j}}{\longrightarrow} \tilde{H} \stackrel{i}{\longrightarrow} \tilde{G} \stackrel{p_{i}}{\longrightarrow} G_{i}
$$

by $\phi_{i}$ and $\phi_{i j}$ respectively. For each $i$, define $m_{i}$ to be the largest integer such that $\phi_{i}^{*}: P\left(G_{i}\right) \rightarrow P(\tilde{H})$ is nontrivial in degree $m_{i}$ (such integer exists because $\phi_{i}^{*}$ is nontrivial, say, in degree 3). Call $G_{i}$ of type 1 if $m_{i}=n\left(G_{i}\right)$, of type 2 if $m_{i}<n\left(G_{i}\right)$. Let us remind the readers once more that the sequence

$$
0 \longrightarrow P(M) \stackrel{\pi^{*}}{\longrightarrow} P(\tilde{G}) \stackrel{i^{*}}{\longrightarrow} P(\tilde{H}) \longrightarrow 0
$$


is exact, that the irreducibility of the action implies that $\phi_{i}^{*}: P\left(G_{i}\right) \rightarrow P(\tilde{H})$ is never a monomorphism for each $i$, and so in particular that $n\left(G_{i}\right) \geqq l(M)$ for each $i$. Let $m$ be the largest integer such that $m<l(M)$ and $m \equiv-1 \bmod 4$. We have $\operatorname{dim} P_{m}\left(G_{i}\right)$ $\geqq 1$ for each $i$. This follows from the observation that the primitive generators of a classical group $G$ always contains an unbroken string $3,7, \ldots$, up to $n(G)$ or $n(G)-2\left(\operatorname{dim} P_{m}\left(G_{i}\right)>1\right.$ may occur if $G_{i}$ belongs to $D$ series). Since $i^{*}: P(\tilde{G}) \rightarrow P(\tilde{H})$ is an isomorphism in degree $m$, we must have $n\left(H_{i}\right) \geqq m$ for each $i$ for otherwise we would have $\operatorname{dim} P_{m}(\tilde{H})<s \leqq \operatorname{dim} P_{m}(\tilde{G})$. In other words, we have $n\left(H_{i}\right) \geqq l(M)-4$ $>u(M)+3 / 2$ by the definition of $m$ and our condition that $2 l(M)>u(M)+11$.

For each $G_{i}$, associate a factor $H_{j}$ with it as follows. Since $\phi_{i}^{*}: P\left(G_{i}\right) \rightarrow P(\tilde{H})$ is nontrivial in degree $m_{i}$, there is some $j$ such that $\phi_{i j}^{*}: P\left(G_{i}\right) \rightarrow P\left(H_{j}\right)$ is nontrivial in degree $m_{i}$. There is only one such $H_{j}$. In fact, suppose $j^{\prime} \neq j$ is such that

$$
\phi_{i j^{\prime}}^{*}: P\left(G_{i}\right) \rightarrow P\left(H_{j^{\prime}}\right)
$$

is nontrivial in degree $m_{i}$. If $G_{i}$ is of type 1 , we must have $n\left(H_{j}\right)=n\left(H_{j^{\prime}}\right)=n\left(G_{i}\right)$ by Lemma 2.6. In particular, $n\left(H_{j}\right)+n\left(H_{j^{\prime}}\right)=2 n\left(G_{i}\right)>n\left(G_{i}\right)+3$. But this would contradict Lemma 2.7 since both $\phi_{i j}$ and $\phi_{i j^{\prime}}$ are certainly nontrivial. If on the other hand $G_{i}$ is of type 2 , then we have $l(M) \leqq n\left(G_{i}\right) \leqq u(M)$. By what we have said above, $n\left(H_{j}\right)+n\left(H_{j^{\prime}}\right)>u(M)+3 \geqq n\left(G_{i}\right)+3$, again contradicts Lemma 2.7. The above association thus defines a map $\alpha:[1, s] \rightarrow[1, s]([1, s]$ is the set of integers from 1 to $s)$. We claim that $\phi_{i j}: H_{j} \rightarrow G_{i}$ is nontrivial if and only if $j=\alpha(i)$. We know that $\phi_{i, \alpha(i)}$ is nontrivial by construction. If $j \neq \alpha(i)$, we wish to show that $\phi_{i j}$ is trivial. If $G_{i}$ is of type 1 , this follows from the relation $n\left(H_{\alpha(i)}\right)+n\left(H_{j}\right)=n\left(G_{i}\right)+n\left(H_{j}\right)$ $>n\left(G_{i}\right)+3$. If $G_{i}$ is of type 2 so that $n\left(G_{i}\right) \leqq u(M)$, this follows again because $n\left(H_{\alpha(i)}\right)+n\left(H_{j}\right)>u(M)+3 \geqq n\left(G_{i}\right)+3$. To finish the proof of Theorem 2.1, we only need to observe that $\alpha$ is onto. For given $H_{j}$, there is at least some $G_{i}$ such that $\phi_{i j}: H_{j} \rightarrow G_{i}$ is nontrivial otherwise $i: \widetilde{H} \rightarrow \widetilde{G}$ will not be an imbedding. The above characterization of $\alpha$ then gives $j=\alpha(i)$. It follows that $\alpha:[1, s] \rightarrow[1, s]$ is a one-toone correspondence. Rearranging indices, we may assume that $\alpha(i)=i$ and what we have shown is that the "matrix" $\left(\phi_{i j}\right)$ is diagonal. This completes the proof of Theorem 2.1.

Making necessary modifications with due care, one can proceed to establish a corresponding decomposition theorem for the specific case of $V_{n, 2 l}$. We shall therefore simply summarize all the cases by stating

THEOREM 2.8. The decomposition Theorem 2.1 applies to the following cases:

(i) $V_{n, k}$, with $k>29$ and $2 k>n+3$,

(ii) $W_{n, k}$, with $k>29$ and $2 k>n+4$,

(iii) $X_{n, k}$, with $k>14$ and $2 k>n+1$.

3. Some nondecomposition theorems for the Stiefel manifolds. We now move on to show that for the spaces $V_{n, k}, W_{n, k}$, and $X_{n, k}$, the kind of decomposition as stated in Theorem 2.1 cannot exist. This would of course yield the conclusion that 
any group $G$ acting transitively and irreducibly on these spaces must be simple. We propose to approach this problem by means of the Steenrod square operations. Unfortunately, the strength of this method is quite limited. As a consequence, our results in this section are rather incomplete. Only in the special case when $k=n-2$, are we able to provide a more satisfactory answer using other topological means. Information of the mod 2 cohomology of the Stiefel manifolds and their square operations are given as follows [3].

(3.1) If $2 k \geqq n, H^{*}\left(V_{n, k} ; Z_{2}\right)$ is an exterior algebra with generators $v_{j}, j=k$, $k+1, \ldots, n-1, \operatorname{deg} v_{j}=j$, and

$$
\begin{aligned}
\mathrm{Sq}^{i} v_{j} & =\left(\begin{array}{l}
j \\
i
\end{array}\right) v_{j+i}, & & i \leqq j, j+i \leqq n-1 \\
& =0, & & \text { otherwise. }
\end{aligned}
$$

(3.2) $H^{*}\left(W_{n, k} ; Z_{2}\right)$ is always an exterior algebra with generators $w_{2 j+1}, j=k$, $k+1, \ldots, n-1, \operatorname{deg} w_{2 j+1}=2 j+1$, and

$$
\begin{aligned}
\mathrm{Sq}^{2 i} w_{2 j+1} & =\left(\begin{array}{l}
j \\
i
\end{array}\right) w_{2(j+i)+1}, & & i \leqq j, j+i \leqq n-1, \\
& =0, & & \text { otherwise. }
\end{aligned}
$$

(3.3) $H^{*}\left(X_{n, k} ; Z_{2}\right)$ is always an exterior algebra with generators $x_{4 j+3}, j=k$, $k+1, \ldots, n-1, \operatorname{deg} x_{4 j+3}=4 j+3$, and

$$
\begin{aligned}
\mathrm{Sq}^{4 i} x_{4 j+3} & =\left(\begin{array}{l}
j \\
i
\end{array}\right) x_{4(j+1)+3}, & & i \leqq j, j+i \leqq n-1, \\
& =0, & & \text { otherwise. }
\end{aligned}
$$

In the above,

$$
\left(\begin{array}{l}
j \\
i
\end{array}\right)
$$

as usual denotes the mod 2 combinatorial coefficient. The square operations in $W_{n, k}$ and $X_{n, k}$ are not given explicitly in [3]. We only remark that formula (3.1) is derived in [3] by passing to the Stiefel-Whitney classes. Using the same computation with the Stiefel-Whitney classes replaced by the Chern classes and the symplectic characteristic classes, one can see that the formulas given in (3.2) and (3.3) are correct.

To illustrate our method, let us first state

Proposition 3.4. Suppose that $2 k \geqq n$, and suppose that both $n$ and $k$ are odd. Then $V_{n, k}$ cannot be decomposed into a product of two manifolds with positive dimensions.

Proof. Suppose $V_{n, k}=M \times N$, then $H^{*}\left(V_{n, k} ; Z_{2}\right)=H^{*}\left(M ; Z_{2}\right) \otimes H^{*}\left(N ; Z_{2}\right)$. We identify $H^{*}\left(M ; Z_{2}\right)$ and $H^{*}\left(N ; Z_{2}\right)$ with the subalgebras $H^{*}\left(M ; Z_{2}\right) \otimes 1$ and $1 \otimes H^{*}\left(N ; Z_{2}\right)$ of $H^{*}\left(V_{n, k} ; Z_{2}\right)$ respectively. Clearly, these subalgebras are closed 
under square operations. Using the condition $2 k \geqq n$, one concludes easily that each $v_{j}, k \leqq j \leqq n-1$, is either in $H^{*}\left(M ; Z_{2}\right)$ or in $H^{*}\left(N ; Z_{2}\right)$. Suppose

$$
v_{k} \in H^{*}\left(M ; Z_{2}\right) \text {. }
$$

Let $l$ be the largest integer such that $v_{j} \in H^{*}\left(M ; Z_{2}\right)$ for all $k \leqq j \leqq l$. We must have $l<n-1$ because $N$ is of positive dimension. We must also have that $l$ is even because otherwise

$$
\left(\begin{array}{l}
l \\
1
\end{array}\right)=1
$$

and we will have $v_{l+1}=\operatorname{Sq}^{1} v_{l} \in H^{*}\left(M ; Z_{2}\right)$, contradicts the definition of $l$. Since both $k$ and $n$ are odd, we have $k+1 \leqq l \leqq n-3$. The situation is thus: we have $v_{l-1}, v_{l} \in H^{*}\left(M ; Z_{2}\right)$ and $v_{l+1}, v_{l+2} \in H^{*}\left(N ; Z_{2}\right)$ (since $\left.v_{l+2}=\mathrm{Sq}^{1} v_{l+1}\right)$. Now it is easy to obtain a contradiction as follows. If $l \equiv 2 \bmod 4$,

$$
\left(\begin{array}{l}
l \\
2
\end{array}\right)=1
$$

and we would have $\mathrm{Sq}^{2} v_{l}=v_{l+2} \in H^{*}\left(M ; Z_{2}\right)$. If $l \equiv 0 \bmod 4$,

$$
\left(\begin{array}{c}
l-1 \\
2
\end{array}\right)=1
$$

and we would have $\mathrm{Sq}^{2} v_{l-1}=v_{l+1} \in H^{*}\left(M ; Z_{2}\right)$. This completes the proof.

For other cases, say $k$ is odd and $n$ is even. The above argument may break down when $l=n-2$ because we can no longer consider $\mathrm{Sq}^{2} v_{l}$ and we may have

$$
\left(\begin{array}{c}
l-1 \\
2
\end{array}\right)=\left(\begin{array}{c}
n-3 \\
2
\end{array}\right)=0 \text {. }
$$

This could be prevented if we assume $n \equiv 2 \bmod 4$. Similarly if $k$ is even, we have to guard against the possibility that $l=k$. Taking into account all these considerations, we can state the following:

THEOREM 3.5. Suppose that $2 k \geqq n, n \neq 00 \bmod 4, k \neq 0 \bmod 4$, and $n-k>2$. Then none of these spaces $V_{n, k}, W_{n, k}$, and $X_{n, k}$ can be decomposed into a product of two manifolds with positive dimensions.

It is obvious that the nondecomposition theorem stated above leaves much to be desired. Within the framework of square operations, one can improve it by imposing conditions on the dyadic expansions of $n$ and $k$. As the gain will not be substantial, we shall be content with the above version and direct our attention to the case $k=n-2$. Here not only can a much better result be obtained, but it also gives some insight to the problem in general.

THEOREM 3.6. None of the Stiefel manifolds $V_{n, n-2}, W_{n, n-2}$, and $X_{n, n-2}$ can be 
decomposed into a product of two homogeneous spaces with positive dimensions unless $n=4,8$ in the real case, $n=2,4$ in the complex case, and $n=1,2$ in the symplectic case.

Proof. Take the real case. We may of course assume that $n$ is even $\geq 4$ ( $n=2$ is of no interest and $\mathrm{Sq}^{1}$ suffices to handle the case when $n$ is odd). Recall that

$$
H_{n-2}\left(V_{n, n-2} ; Z\right)=H_{n-1}\left(V_{n, n-2} ; Z\right)=H_{2 n-3}\left(V_{n, n-2} ; Z\right)=Z
$$

( $Z$ is the group of integers), zero in other positive dimensions, and that $V_{n, n-2}$ is $(n-3)$-connected. If $V_{n, n-2}=M \times N$ is a product of two homogeneous spaces, one can easily deduce that $M$ is an integral homology $(n-2)$-sphere and $N$ is an integral homology $(n-1)$-sphere. Being homogeneous spaces and simply connected, they must be actual spheres [4]. Moreover, it is also true that $p_{*}: H_{*}\left(V_{n, n-2} ; Z\right) \rightarrow H_{*}(M ; Z)$ induced by the projection $p: V_{n, n-2} \rightarrow M$ is an isomorphism in dimension zero and $n-2$. According to Dold [7], this means the fibration $S^{n-2} \rightarrow V_{n, n-2} \rightarrow S^{n-1}$ is fiber-homotopically trivial. But then a theorem of Milnor and Spanier [9] says that this could be so only if $n=2,4$ or 8 . For the complex and the symplectic cases, one proceeds in the same manner. The only observations needed are the facts that (i) the sphere bundle $S^{2 n-3} \rightarrow W_{n, n-2} \rightarrow S^{2 n-1}$ joined with a trivial zero sphere bundle becomes the bundle $S^{2 n-2} \rightarrow V_{2 n, 2 n-2} \rightarrow S^{2 n-1}$ and (ii) the sphere bundle $S^{4 n-5} \rightarrow X_{n, n-2} \rightarrow S^{4 n-1}$ joined with a trivial 1-sphere bundle becomes the bundle $S^{4 n-3} \rightarrow W_{2 n, 2 n-2} \rightarrow S^{4 n-1}$ (translate into vector bundle and Whitney sum, if one likes).

For the general case, it seems now quite natural that one should try to decide whether the fibration $V_{n-1, k} \rightarrow V_{n, k} \rightarrow S^{n-1}$ is fiber-homotopically trivial or not. If one recalls that the theorem of Milnor and Spanier quoted above is based on Adams' work on Hopf invariant, one can perhaps have some idea about what might be involved. At the present time, the authors have no answer to this question, but we hope to come back to it later.

4. Main theorems. We are now ready to present the main theorems of this paper. It is apparent by now that we can treat only some of the Stiefel manifolds, so let us first summarize all conditions to make all the theorems of the previous sections applicable. We shall ask that the pair $(n, k)$ satisfies the condition below.

Condition 4.1. (i) $k \leqq n-2$ and $2 k>n+4$, (ii) $k>29$ and (iii) in case $k<n-2$, then $k \not \equiv 0 \bmod 4$ and $n \not \equiv 0 \bmod 4$.

THEOREM 4.2. In the range when $(n, k)$ satisfies Condition 4.1 , the standard actions $\left(\mathrm{SO}(n), \mathrm{SO}(k) ; V_{n, k}\right),\left(\mathrm{SU}(n), \mathrm{SU}(k) ; W_{n, k}\right)$ and $\left(\mathrm{Sp}(n), \mathrm{Sp}(k) ; X_{n, k}\right)$ are the only effective and irreducible transitive actions on $V_{n, k}, W_{n, k}$ and $X_{n, k}$ respectively.

Proof. For definiteness, let us consider the case $V_{n, k}$ with both $n$ and $k$ odd, say $n=2 m+1$ and $k=2 l+1$. Let $\left(G, H ; V_{n, k}\right)$ be an arbitrary effective and irreducible transitive action on $V_{n, k}$. We have to show that $G=\mathrm{SO}(n), H=\mathrm{SO}(k)$, and 
$\mathrm{SO}(k) \subset \mathrm{SO}(n)$ is (conjugate to) the standard imbedding. Let $\left(\tilde{G}, \tilde{H} ; V_{n, k}\right)$ $\sim\left(G, H ; V_{n, k}\right)$ be the lifted action and $N \subset \tilde{G}$ the finite normal subgroup such that $\tilde{G} / N=G$. In view of Condition 4.1 and earlier results, we are guaranteed that both $\tilde{G}$ and $\tilde{H}$ are simple, simply connected classical groups. Referring back to the proof of Theorem 2.1, we can see easily that $P(\widetilde{G})=(3,7, \ldots, 4 m-1)$ and $P(\tilde{H})=(3,7$, $\ldots, 4 l-1)$. So the possible candidates for $\widetilde{G}$ are $\operatorname{Spin}(2 m+1)$ and $\operatorname{Sp}(m)$, and the possible candidates for $\tilde{H}$ are $\operatorname{Spin}(2 l+1)$ and $\operatorname{Sp}(l)$. Two of the cases, namely $\mathrm{Sp}(l) \subset \mathrm{Spin}(2 m+1)$ and $\mathrm{Spin}(2 l+1) \subset \mathrm{Sp}(m)$, are eliminated by (2.3) and (2.4) because the range $2(2 l+1)>(2 m+1)+4$ is not right for nontrivial imbedding. The possibility $\mathrm{Sp}(l) \subset \mathrm{Sp}(m)$ is also out. Because in this case, the imbedding must be standard by (2.4), giving $V_{2 m+1,2 l+1}=X_{m, l}$. But this is clearly impossible because, for example, $H^{*}\left(X_{m, l} ; Z\right)$ has no torsion (remember that $m-l \geqq 2$ ). Thus we have $\tilde{G}=\operatorname{Spin}(2 m+1), \tilde{H}=\operatorname{Spin}(2 l+1)$ and $\tilde{H}=\operatorname{Spin}(2 l+1) \subset \operatorname{Spin}(2 m+1)=\widetilde{G}$ is standard. Let $Z_{2} \subset \operatorname{Spin}(2 m+1)$ be the center. We have $Z_{2} \subset \widetilde{H}$ and therefore $Z_{2} \subset N$. If $Z_{2} \neq N$, the action of $\widetilde{G} / Z_{2}$ will not be effective. On the other hand, we have just seen that $\tilde{G} / Z_{2}=\operatorname{SO}(2 m+1)$ acts on $V_{2 m+1,2 l+1}$ in the standard way which is effective. Hence $Z_{2}=N$ and the proof is complete. The argument for other cases are all similar.

Turning now to the general case where the action is not necessarily irreducible. Recall the example mentioned in the introduction, that is, the action of $\operatorname{SO}(n)$ $\times \mathrm{SO}(n-k)$ on $V_{n, k}$ via left and right matrix multiplication. More generally, we can restrict it to a subgroup $G$ with $\mathrm{SO}(n) \subset G \subset \mathrm{SO}(n) \times \mathrm{SO}(n-k)$ and this is still transitive on $V_{n, k}$. This action may fail to be effective but is always "almost effective", that is, the normal subgroup acting as identity homeomorphism is a finite group. In our case, it is at most a group $Z_{2}$, diagonally imbedded in $\operatorname{SO}(n)$ $\times \mathrm{SO}(n-k)$ as $(-1,-1)$. We shall denote it by $K_{n, k} \subset \mathrm{SO}(n) \times \mathrm{SO}(n-k)$. Let us look at the action of $\mathrm{SO}(n) \times \mathrm{SO}(n-k)$ on $V_{n, k}$ more closely. If we go up to $\operatorname{Spin}(n)$ $\times \operatorname{Spin}(n-k)$, we see that the isotropy subgroup consists of those elements of the form $\left(g_{1} g_{2}, g_{2}\right), g_{1} \in \operatorname{Spin}(k), g_{2} \in \operatorname{Spin}(n-k)$ (as a group, it is therefore isomorphic to $\operatorname{Spin}(k) \times \operatorname{Spin}(n-k))$. This enables us to identify the action of $\operatorname{Spin}(n) \times \operatorname{Spin}(n-k)$ on $V_{n, k}$. There are of course complex and symplectic analogues. Namely $\mathrm{SU}(n) \times \mathrm{SU}(n-k)$ acting on $W_{n, k}$ and $\mathrm{Sp}(n) \times \mathrm{Sp}(n-k)$ on $X_{n, k}$. In each case, the noneffective part is still at most a finite group to be denoted indiscriminately by $K_{n, k}$.

THEOREM 4.3. Let $G$ be a compact connected Lie group acting transitively and effectively on $V_{n, k}$. If $(n, k)$ satisfies 4.1 , then $\mathrm{SO}(n) \subset G \subset \mathrm{SO}(n) \times \mathrm{SO}(n-k) / K_{n, k}$ and the action is the restriction on $G$ of the standard action of $\mathrm{SO}(n) \times \mathrm{SO}(n-k) / K_{n, k}$ on $V_{n, k}$. Corresponding statements are also true in the complex and the symplectic cases, with $V_{n, k}$ replaced by $W_{n, k}, X_{n, k}$, and $\mathrm{SO}(n) \times \mathrm{SO}(n-k) / K_{n, k}$ replaced by $\mathrm{SU}(n) \times \mathrm{SU}(n-k) / K_{n, k}, \mathrm{Sp}(n) \times \mathrm{Sp}(n-k) / K_{n, k}$ respectively.

Proof. We shall again only consider the real case with $n$ and $k$ both odd (so that 
$\left.K_{n, k}=1\right)$. Let $\left(G, H, V_{n, k}\right)$ be an effective action and $\left(\tilde{G}, \tilde{H} ; V_{n, k}\right) \sim\left(G, H ; V_{n, k}\right)$ the lifted action with $G=\tilde{G} / N$. It is easy to see that $\tilde{G}=G_{1} \times G_{2}$ where $G_{1}$ acts transitively and irreducibly on $V_{n, k}$. From the proof of the previous theorem, we can see that $G_{1}=\operatorname{Spin}(n)$ and it acts on $V_{n, k}$ via standard action. Let $i: \tilde{H} \rightarrow \tilde{G}$, $i_{1}: G_{1} \rightarrow \tilde{G}, i_{2}: G_{2} \rightarrow \widetilde{G}$ be inclusions and $p_{1}: \tilde{G} \rightarrow G_{1}, p_{2}: \widetilde{G} \rightarrow G_{2}$ projections. Define $H_{1}=i_{1}^{-1}(\tilde{H}), H_{2}=i_{2}^{-1}(\tilde{H})$ and $\Gamma_{1}=\operatorname{Im} p_{1} \circ i, \Gamma_{2}=\operatorname{Im} p_{2} \circ i$. The following is easily verified: (i) $H_{1} \subset \Gamma_{1} \subset G_{1}, H_{2} \subset \Gamma_{2} \subset G_{2}, H_{1}$ is normal in $\Gamma_{1}, H_{2}$ is normal in $\Gamma_{2}$. (ii) $H_{1} \times H_{2} \subset \tilde{H} \subset \Gamma_{1} \times \Gamma_{2}$ and (iii) the inclusions $\Gamma_{i} \rightarrow \Gamma_{1} \times \Gamma_{2}, i=1,2$, induce homeomorphisms $\Gamma_{i} / H_{i} \rightarrow \Gamma_{1} \times \Gamma_{2} / \tilde{H}, i=1,2$ (see [10]). In particular this yields a group isomorphism $\phi: \Gamma_{2} / H_{2} \rightarrow \Gamma_{1} / H_{1}$ given as follows. Take $\tau_{2} H_{2} \in \Gamma_{2} / H_{2}$, choose $\tau_{1} \in \Gamma_{1}$ so that $\left(\tau_{1}, \tau_{2}\right) \in \tilde{H}$, then $\phi\left(\tau_{2} H_{2}\right)=\tau_{1} H_{1}$. Now we claim that $\Gamma_{2}=G_{2}$. To see this we use the diagram of Proposition 1.4 by taking $G_{0}=G_{1}$. As $G_{1}$ acts transitively on $V_{n, k}, P\left(V_{n, k}\right) \rightarrow P\left(G_{1}\right)$ is a monomorphism. Hence $i^{*} \circ p_{2}^{*}: P\left(G_{2}\right) \rightarrow P(\tilde{H})$ is also a monomorphism. As we have seen before, this implies that $p_{2} \circ i: \tilde{H} \rightarrow G_{2}$ is an epimorphism, which is precisely what we asserted. It follows that $H_{2}$ is normal in $G_{2}$ and therefore normal in $\tilde{G}$. Since the action of $\tilde{G}$ is almost effective, we must have $\mathrm{H}_{2} \subset \mathrm{N}$ is a finite group. We might as well assume (and we will do so) that $H_{2}$ is trivial for otherwise we can simply factor it out from $\tilde{G}$. Next we have $H_{1}=\operatorname{Spin}(k) \subset \Gamma_{1} \subset \operatorname{Spin}(n)=G$. As $H_{1}$ is normal in $\Gamma_{1}$ and $\Gamma_{1}$ is connected ( $\tilde{H}$ is connected), $\Gamma_{1}$ is contained in the identity component of the normalizer of $\operatorname{Spin}(k)$ in $\operatorname{Spin}(n)$, which is $\operatorname{Spin}(k) \times \operatorname{Spin}(n-k)$. Therefore the isomorphism $\phi: G_{2}=\Gamma_{2} \rightarrow \Gamma_{1} / H_{1}=\operatorname{Spin}(n-k)$ imbeds $G_{2}$ as a subgroup of $\operatorname{Spin}(n-k)$. So far, we have shown that $\tilde{G}$ is a subgroup of $\operatorname{Spin}(n) \times \operatorname{Spin}(n-k)$. We will now look at $\tilde{H}$. Take $\left(g_{1}, g_{2}\right) \in \tilde{H}$. Since $g_{1} \in \Gamma_{1}, g_{1}=h k, h \in \operatorname{Spin}(k)$, $k \in \operatorname{Spin}(n-k)$. Now $\tilde{H}$ goes into $\operatorname{Spin}(n) \times \operatorname{Spin}(n-k)$ via $1 \times \phi$. Since $\left(g_{1}, g_{2}\right) \in \tilde{H}$ and $g_{1} H_{1}=k$, we have $\phi\left(g_{2}\right)=k$. That is, $\tilde{H}$ goes precisely to the subgroup of $\operatorname{Spin}(k) \times \operatorname{Spin}(n-k)$ of elements of the form $(h k, k), h \in \operatorname{Spin}(k), k \in \operatorname{Spin}(n-k)$. Just as in Theorem 4.2, we can then finish the proof by showing that $N$ also goes precisely to the right place.

Finally, we remark that throughout the whole paper, the only place where topological properties of the Stiefel manifolds beyond their homotopy and homology are used is Theorem 3.6. Therefore if we modify Condition 4.1 by adding (iv) in case $k=n-2$, then $n$ is odd, and call this Condition $(4.1)^{\prime}$, we can state

THEOREM 4.4. Let $M$ be a homogeneous space with the same homotopy type of $V_{n, k}, W_{n, k}$, or $X_{n, k}$. If the pair $(n, k)$ satisfies Condition $(4.1)^{\prime}$, then $M$ is actually diffeomorphic to $V_{n, k}, W_{n, k}$, or $X_{n, k}$ respectively.

\section{REFERENCES}

1. A. Borel, Some remarks about Lie groups transitive on spheres and tori, Bull. Amer. Math. Soc. 55 (1949), 580-587.

2. - Sur la cohomologie des espaces fibrés principaux et des espaces homogènes de groupes de Lie compacts, Ann. of Math. (2) 57 (1953), 115-207. 
3. - La cohomologie mod 2 de certains espaces homogènes, Comment. Math. Helv. 27 (1953), 165-197.

4. - Les bouts des espaces homogènes de groupes de Lie, Ann. of Math. 58 (1953), 443-457.

5. A. Borel and F. Hirzebruch, Characteristic classes and homogeneous spaces. I, Amer. J. Math. 80 (1958), 485-538.

6. C. Chevalley, Theory of Lie groups, Princeton Univ. Press, Princeton, N. J., 1946.

7. A. Dold, Uber fasernweise Homotopieäquivalenz von Faserräumen, Math. Z. 62 (1955), 111-136.

8. J. Milnor and J. C. Moore, On the structure of Hopf algebras, Ann. of Math. 81 (1965), 211-264.

9. J. Milnor, J. C. Moore, and E. Spanier, Two remarks on fiber homotopy type, Pacific J. Math. 10 (1960), 585-590.

10. D. Montgomery and H. Samelson, Transformation groups of spheres, Ann. of Math. 44 (1943), 454-470.

11. J. Poncét, Groupes des Lie compacts de transformations de l'espace euclidien et les spheres comme espaces homogènes, Comment. Math. Helv. 33 (1959), 109-120.

BROWN UNIVERSITY,

Providence, Rhode Island

UNIVERSITY OF VIRGINIA,

Charlottesville, Virginia

The INSTITUTE FOR AdVANCEd STUdy,

Princeton, New Jersey 